\title{
EXPANDING THE COMPETITIVE PROFILE MATRIX: INTRODUCING THE PRODUCTION/OPERATIONS MANAGEMENT, MARKETING, HUMAN RESOURCE MANAGEMENT, FINANCE/ACCOUNTING, RESEARCH AND DEVELOPMENT, AND INFORMATION SYSTEMS COMPETITIVE PROFILE MATRICES
}

\author{
Charles J. Capps III \\ Sam Houston State University • Huntsville, TX \\ Christopher M. Cassidy \\ Sam Houston State University • Huntsville, TX \\ Renee Gravois \\ Sam Houston State University • Huntsville, TX \\ Janis A. Warner \\ Sam Houston State University • Huntsville, TX
}

\section{ABSTRACT}

Capps and Glissmeyer (2012) proposed an extension to the Internal Factor Evaluation and External Factor Evaluation matrices that included an Internal Competitive Profile Matrix and an External Competitive Profile Matrix, which use a forced ranking that provides greater understanding of the internal and external categories to which an organization must attend. Cassidy, Glissmeyer, and Capps (2013) mapped an Internal-External (I-E) Matrix using traditional and extended techniques to enable a greater comparative understanding of the relative strengths, weaknesses, opportunities, and threats of respective companies in an analogous Company Comparison Internal-External Matrix using horizontal analysis. This matrix approach to strategic analysis and decision-making is presented in Fred David's Strategic Management, 16th edition (2016). A traditional Competitive Profile Matrix (CPM) is a corporate-level analytic tool that uses critical success factors. This paper offers a conceptual expansion of the CPM to include a Production/Operations Management CPM, Marketing CPM, Human Resource Management CPM, Finance/ Accounting CPM, Research and Development CPM, and an Information Systems CPM to provide additional strategic decision-making analytical tools. If analytical thoroughness is a major goal, then expanding the CPM into the six functional business areas should provide further depth of analysis and more in-depth insight. 
Keywords: strategic decision-making, performance measurement, competitive profile matrix, internal factor evaluation matrix, external factor evaluation matrix, internal-external matrix, strategic decision-making tools

\section{INTRODUCTION}

Many strategic management professors teaching the capstone course of the Bachelor of Business Administration degree use the matrix approach, presented in Fred David's Strategic Management, 16th edition (2016). David uses a basic horizontal approach to strategic analysis and presents three stages of analytical matrices for strategic decision-making. The input stage, which includes the Internal Factor Evaluation Matrix, the External Factor Evaluation Matrix, and the Competitive Profile Matrix (CPM). The matching stage, which includes the Strengths, Weaknesses, Opportunities, and Threats Matrix, the SPACE matrix, the Boston Consulting Group matrix, the Internal-External (I-E) matrix, and the Grand Strategy matrix;. Finally the decision stage, which only includes the Quantitative Strategic Planning (QSPM) Matrix. The analytical flow of the matrices is horizontal, and takes the output from the input stage, and uses the matching stage matrices to produce recommendations for consideration in the decision stage. The analytical flow is horizontal except for one, the CPM. Could the CPM be expanded to extend its analytical use? Could the CPM be made more useful by expanding it vertically? Additionally, many strategic management professors believe the process of strategic analysis is as important as the final recommendations. Assuming the process itself is valuable, then expansion of the CPM vertically in the strategic management analytical decision-making process to include the six functional business areas is beneficial because it is vital to not overlook something important.

Our recent work extended the traditional CPM horizontally by creating an Internal Competitive Profile Matrix and an External Competitive Profile Matrix, which were subsequently plotted on a nine cell Company Comparison InternalExternal Matrix (Capps \& Cassidy, 2016). During the exercise, we considered this question: Are there benefits to using vertical analysis when considering a CPM? We believe the answer is "yes" when the corporate CPM is supported by functional area matrices with the top ten concepts in the six functional business areas (Capps 
\& Cassidy, 2016). Moreover, because strategists believe the depth and thoroughness of the strategic management decision-making analytical process is a fundamental reason for conducting strategic audits, the authors introduce the following six new matrices Production/Operations Management CPM (POM-CPM), Marketing CPM (M-CPM), Finance/Accounting CPM (F-CPM), Human Resource Management CPM (HRM-CPM), Research and Development (R\&D-CPM), and Information Systems CPM (IS-CPM) to provide more in-depth strategic analytical insight when analyzing competing companies.

\section{THE NEW FUNTIONAL LEVEL CPM MATRICES}

Since there is always a need to advance analytical tools used in the strategic management decision-making process (Fleisher \& Bensoussan, 2003, 2007; Chang \& Huang, 2006; Bygrave \& Zacharkis, 2010; Capps \& Glissmeyer, 2012; Cassidy, Glissmeyer, \& Capps, 2013), Capps and Glissmeyer (2012) advanced the strategic management decision-making process by creating the Internal Competitive Profile Matrix and an External Competitive Profile Matrix tools for added insight. Cassidy, Glissmeyer, and Capps (2013) visually mapped an I-E matrix using both traditional and extended concepts. This produced different plotting points, and sometimes the result was also a different I-E cell assignment. Based on this foundation we now shift our focus and address the issue of how a CPM can be expanded using vertical analysis by creating six new functional area matrices: the POM-CPM, M-CPM, HRM-CPM, F-CPM, R\&D-CPM, and an IS-CPM. Using vertical analysis, we introduce six CPM extensions expanding the matrix into the six functional business areas for more depth of analysis of companies' major differences. Below are the six proposed CPM matrices for your consideration. Introduced first is the POM-CPM, which focuses on the top ten areas of Production/Operations Management (POM) needing analysis and comparison in POM. 
Table 1

Introducing a Production/Operations Management Competitive Profile Matrix (POM-CPM) for Four Hypothetical Firms

\begin{tabular}{|c|c|c|c|c|c|c|c|c|c|}
\hline & \multirow[b]{2}{*}{ Weight } & \multicolumn{2}{|c|}{ Company 1} & \multicolumn{2}{|c|}{ Company 2} & \multicolumn{2}{|c|}{ Company 3} & \multicolumn{2}{|c|}{ Company 4} \\
\hline & & Rating & Score & Rating & Score & Rating & Score & Rating & Score \\
\hline Process & 0.10 & 2 & 0.20 & 3 & 0.30 & 1 & 0.10 & 4 & 0.40 \\
\hline Capacity & 0.10 & 2 & 0.20 & 3 & 0.30 & 1 & 0.10 & 4 & 0.40 \\
\hline \% Capacity Used & 0.10 & 1 & 0.10 & 2 & 0.20 & 3 & 0.30 & 4 & 0.40 \\
\hline Workforce & 0.10 & 3 & 0.30 & 1 & 0.10 & 4 & 0.40 & 2 & 0.20 \\
\hline Automation Level & 0.10 & 1 & 0.10 & 3 & 0.30 & 2 & 0.20 & 4 & 0.40 \\
\hline $\begin{array}{l}\text { Just-in-Time } \\
\text { Inventory }\end{array}$ & 0.10 & 1 & 0.10 & 4 & 0.40 & 2 & 0.20 & 3 & 0.30 \\
\hline $\begin{array}{l}\text { Total Quality } \\
\text { Management } \\
\text { Quality Control }\end{array}$ & 0.10 & 4 & 0.40 & 3 & 0.30 & 2 & 0.20 & 1 & 0.10 \\
\hline $\begin{array}{l}\text { Customer Tech } \\
\text { Support }\end{array}$ & 0.10 & 1 & 0.10 & 3 & 0.30 & 4 & 0.40 & 2 & 0.20 \\
\hline Innovation & 0.10 & 3 & 0.30 & 2 & 0.20 & 1 & 0.10 & 4 & 0.40 \\
\hline Infrastructure & 0.10 & 2 & 0.20 & 3 & 0.30 & 1 & 0.10 & 4 & 0.40 \\
\hline Totals & 1.00 & - & 2.00 & - & 2.70 & - & 2.10 & - & 3.20 \\
\hline
\end{tabular}


Second, the Marketing Competitive Profile Matrix (M-CPM) is introduced with the ten major marketing concepts that need monitoring for comparison analysis in marketing.

Table 2

Introducing a Marketing Competitive Profile Matrix (M-CPM) for Four Hypothetical Firms

\begin{tabular}{|c|c|c|c|c|c|c|c|c|c|}
\hline & \multirow[b]{2}{*}{ Weight } & \multicolumn{2}{|c|}{ Company 1} & \multicolumn{2}{|c|}{ Company 2} & \multicolumn{2}{|c|}{ Company 3} & \multicolumn{2}{|c|}{ Company 4} \\
\hline & & Rating & Score & Rating & Score & Rating & Score & Rating & Score \\
\hline Market Share & 0.10 & 1 & 0.10 & 3 & 0.30 & 2 & 0.20 & 4 & 0.40 \\
\hline Advertising & 0.10 & 1 & 0.10 & 2 & 0.20 & 3 & 0.30 & 4 & 0.40 \\
\hline Inventory Turnover & 0.10 & 3 & 0.30 & 2 & 0.20 & 1 & 0.10 & 4 & 0.40 \\
\hline Customer Analysis & 0.10 & 1 & 0.10 & 4 & 0.40 & 2 & 0.20 & 3 & 0.30 \\
\hline $\begin{array}{l}\text { Opportunity } \\
\text { Analysis }\end{array}$ & 0.10 & 2 & 0.20 & 3 & 0.30 & 4 & 0.40 & 1 & 0.10 \\
\hline Marketing Research & 0.10 & 1 & 0.10 & 2 & 0.20 & 3 & 0.30 & 4 & 0.40 \\
\hline $\begin{array}{l}\text { Selling Products } \\
\text { and Services }\end{array}$ & 0.10 & 1 & 0.10 & 3 & 0.30 & 4 & 0.40 & 2 & 0.20 \\
\hline $\begin{array}{l}\text { Product and } \\
\text { Service Planning }\end{array}$ & 0.10 & 2 & 0.20 & 3 & 0.30 & 1 & 0.10 & 4 & 0.40 \\
\hline Distribution & 0.10 & 3 & 0.30 & 1 & 0.10 & 4 & 0.40 & 2 & 0.20 \\
\hline Pricing & 0.10 & 2 & 0.20 & 3 & 0.30 & 1 & 0.10 & 4 & 0.40 \\
\hline Totals & 1.00 & - & 1.70 & - & 2.60 & - & 2.50 & - & 3.20 \\
\hline
\end{tabular}


Third, we introduce the Human Resource Management Competitive Profile Matrix (HRM- CPM) with the ten primary human resources management concepts that need monitoring for HRM comparative analysis.

Table 3

Introducing a Human Resource Management Competitive Profile Matrix (HRM-CPM) for Four Hypothetical Firms

\begin{tabular}{|c|c|c|c|c|c|c|c|c|c|}
\hline & \multirow[b]{2}{*}{ Weight } & \multicolumn{2}{|c|}{ Company 1} & \multicolumn{2}{|c|}{ Company 2} & \multicolumn{2}{|c|}{ Company 3} & \multicolumn{2}{|c|}{ Company 4} \\
\hline & & Rating & Score & Rating & Score & Rating & Score & Rating & Score \\
\hline $\begin{array}{l}\text { Revenue Per } \\
\text { Employee }\end{array}$ & 0.10 & 1 & 0.10 & 2 & 0.20 & 3 & 0.30 & 4 & 0.40 \\
\hline $\begin{array}{l}\text { Compensation Per } \\
\text { Employee }\end{array}$ & 0.10 & 2 & 0.20 & 3 & 0.30 & 1 & 0.10 & 4 & 0.40 \\
\hline $\begin{array}{l}\text { Training Cost } \\
\text { Per Employee }\end{array}$ & 0.10 & 3 & 0.30 & 1 & 0.10 & 4 & 0.40 & 2 & 0.20 \\
\hline $\begin{array}{l}\text { Employee } \\
\text { Absenteeism Rate }\end{array}$ & 0.10 & 1 & 0.10 & 4 & 0.40 & 2 & 0.20 & 3 & 0.30 \\
\hline $\begin{array}{l}\text { Employee } \\
\text { Turnover Rate }\end{array}$ & 0.10 & 4 & 0.40 & 3 & 0.30 & 2 & 0.20 & 1 & 0.10 \\
\hline $\begin{array}{l}\text { OSHA Complaints } \\
\text { Rate }\end{array}$ & 0.10 & 1 & 0.10 & 3 & 0.30 & 2 & 0.20 & 4 & 0.40 \\
\hline $\begin{array}{l}\text { EEOC Complaint } \\
\text { Rate }\end{array}$ & 0.10 & 1 & 0.10 & 3 & 0.30 & 4 & 0.40 & 2 & 0.20 \\
\hline $\begin{array}{l}\text { Grievance and } \\
\text { Lawsuits Rate }\end{array}$ & 0.10 & 3 & 0.30 & 2 & 0.20 & 1 & 0.10 & 4 & 0.40 \\
\hline $\begin{array}{l}\text { Core Employee's } \\
\text { Experience }\end{array}$ & 0.10 & 2 & 0.20 & 3 & 0.30 & 1 & 0.10 & 4 & 0.40 \\
\hline $\begin{array}{l}\text { \% Contingent } \\
\text { Employees }\end{array}$ & 0.10 & 2 & 0.20 & 3 & 0.30 & 1 & 0.10 & 4 & 0.40 \\
\hline Totals & 1.00 & - & 2.00 & - & 2.70 & - & 2.10 & - & 3.20 \\
\hline
\end{tabular}

NOTE: OSHA = U.S. Occupational Safety and Health Administration; EEOC = U.S. Equal Employment Opportunity Commission 
Fourth, the Finance/Accounting Competitive Profile Matrix (F-CPM) is introduced with its ten primary financial concepts that need monitoring for comparison analysis in finance and accounting.

Table 4

Introducing a Financial Competitive Profile Matrix (F-CPM) for Four Hypothetical Firms

\begin{tabular}{|c|c|c|c|c|c|c|c|c|c|}
\hline & \multirow[b]{2}{*}{ Weight } & \multicolumn{2}{|c|}{ Company 1} & \multicolumn{2}{|c|}{ Company 2} & \multicolumn{2}{|c|}{ Company 3} & \multicolumn{2}{|c|}{ Company 4} \\
\hline & & Rating & Score & Rating & Score & Rating & Score & Rating & Score \\
\hline Revenue & 0.10 & 1 & 0.10 & 2 & 0.20 & 3 & 0.30 & 4 & 0.40 \\
\hline Profit Margin & 0.10 & 2 & 0.20 & 3 & 0.30 & 1 & 0.10 & 4 & 0.40 \\
\hline Quick Ratio & 0.10 & 4 & 0.40 & 3 & 0.30 & 2 & 0.20 & 1 & 0.10 \\
\hline Current Ratio & 0.10 & 3 & 0.30 & 2 & 0.20 & 1 & 0.10 & 4 & 0.40 \\
\hline $\begin{array}{l}\text { Return on } \\
\text { Investment }\end{array}$ & 0.10 & 2 & 0.20 & 3 & 0.30 & 1 & 0.10 & 4 & 0.40 \\
\hline Return on Equity & 0.10 & 2 & 0.20 & 3 & 0.30 & 1 & 0.10 & 4 & 0.40 \\
\hline Return on Assets & 0.10 & 3 & 0.30 & 1 & 0.10 & 4 & 0.40 & 2 & 0.20 \\
\hline Earnings per Share & 0.10 & 1 & 0.10 & 3 & 0.30 & 2 & 0.20 & 4 & 0.40 \\
\hline $\begin{array}{l}\text { Debt-to-Equity } \\
\text { Ratio }\end{array}$ & 0.10 & 1 & 0.10 & 4 & 0.40 & 2 & 0.20 & 3 & 0.30 \\
\hline $\begin{array}{l}\text { Market } \\
\text { Capitalization }\end{array}$ & 0.10 & 1 & 0.10 & 3 & 0.30 & 4 & 0.40 & 2 & 0.20 \\
\hline Totals & 1.00 & - & 2.00 & - & 2.70 & - & 2.10 & - & 3.20 \\
\hline
\end{tabular}


Fifth, we introduce the Research and Development Competitive Profile Matrix (R\&D-CPM) with the top ten research and development concepts that need monitoring for R\&D comparative analysis.

Table 5

Introducing a Research and Development Management Competitive Profile Matrix (R\&DMCPM) for Four Hypothetical Firms

\begin{tabular}{|c|c|c|c|c|c|c|c|c|c|}
\hline & \multirow[b]{2}{*}{ Weight } & \multicolumn{2}{|c|}{ Company 1} & \multicolumn{2}{|c|}{ Company 2} & \multicolumn{2}{|c|}{ Company 3} & \multicolumn{2}{|c|}{ Company 4} \\
\hline & & Rating & Score & Rating & Score & Rating & Score & Rating & Score \\
\hline R\&D Investment & 0.10 & 2 & 0.20 & 3 & 0.30 & 1 & 0.10 & 4 & 0.40 \\
\hline $\begin{array}{l}\text { Product or Process } \\
\text { R\&D Focus }\end{array}$ & 0.10 & 1 & 0.10 & 3 & 0.30 & 4 & 0.40 & 2 & 0.20 \\
\hline Internal R\&D Focus & 0.10 & 2 & 0.20 & 3 & 0.30 & 1 & 0.10 & 4 & 0.40 \\
\hline External R\&D Focus & 0.10 & 2 & 0.20 & 3 & 0.30 & 1 & 0.10 & 4 & 0.40 \\
\hline $\begin{array}{l}\text { Both Internal and } \\
\text { External R\&D Focus }\end{array}$ & 0.10 & 1 & 0.10 & 3 & 0.30 & 4 & 0.40 & 2 & 0.20 \\
\hline $\begin{array}{l}\text { First Mover } \\
\text { Advantage }\end{array}$ & 0.10 & 1 & 0.10 & 2 & 0.20 & 3 & 0.30 & 4 & 0.40 \\
\hline Innovative Imitator & 0.10 & 3 & 0.30 & 1 & 0.10 & 4 & 0.40 & 2 & 0.20 \\
\hline Low Cost Producer & 0.10 & 1 & 0.10 & 3 & 0.30 & 2 & 0.20 & 4 & 0.40 \\
\hline $\begin{array}{l}\text { Product } \\
\text { and Process } \\
\text { Total Quality } \\
\text { Management }\end{array}$ & 0.10 & 4 & 0.40 & 3 & 0.30 & 2 & 0.20 & 1 & 0.10 \\
\hline $\begin{array}{l}\text { Basic and Applied } \\
\text { Innovation }\end{array}$ & 0.10 & 3 & 0.30 & 2 & 0.20 & 1 & 0.10 & 4 & 0.40 \\
\hline Totals & 1.00 & - & 2.00 & - & 2.60 & - & 2.30 & - & 3.10 \\
\hline
\end{tabular}


Finally, the Information Systems Competitive Profile Matrix (IS-CPM) is introduced with its ten major information systems concepts that need monitoring for comparative analysis in IS.

Table 6

Introducing an Information Systems Competitive Profile Matrix (IS-CPM) for Four Hypothetical Firms

\begin{tabular}{|c|c|c|c|c|c|c|c|c|c|}
\hline & \multirow[b]{2}{*}{ Weight } & \multicolumn{2}{|c|}{ Company 1} & \multicolumn{2}{|c|}{ Company 2} & \multicolumn{2}{|c|}{ Company 3} & \multicolumn{2}{|c|}{ Company 4} \\
\hline & & Rating & Score & Rating & Score & Rating & Score & Rating & Score \\
\hline $\begin{array}{l}\text { Good Change } \\
\text { Strategy }\end{array}$ & 0.10 & 2 & 0.20 & 3 & 0.30 & 1 & 0.10 & 4 & 0.40 \\
\hline $\begin{array}{l}\text { Access to } \\
\text { Appropriate IT }\end{array}$ & 0.10 & 1 & 0.10 & 3 & 0.30 & 4 & 0.40 & 2 & 0.20 \\
\hline $\begin{array}{l}\text { Technology and IT } \\
\text { Personnel }\end{array}$ & 0.10 & 3 & 0.30 & 1 & 0.10 & 4 & 0.40 & 2 & 0.20 \\
\hline $\begin{array}{l}\text { Adequate } \\
\text { Management } \\
\text { Support / Owner }\end{array}$ & 0.10 & 3 & 0.30 & 1 & 0.10 & 4 & 0.40 & 2 & 0.20 \\
\hline $\begin{array}{l}\text { Effective IT } \\
\text { and Business } \\
\text { Communication }\end{array}$ & 0.10 & 2 & 0.20 & 3 & 0.30 & 1 & 0.10 & 4 & 0.40 \\
\hline $\begin{array}{l}\text { Identify and Select } \\
\text { Project Champion }\end{array}$ & 0.10 & 2 & 0.20 & 3 & 0.30 & 1 & 0.10 & 4 & 0.40 \\
\hline $\begin{array}{l}\text { Alignment of Vision } \\
\text { and Plans between } \\
\text { IT and Business }\end{array}$ & 0.10 & 1 & 0.10 & 3 & 0.30 & 4 & 0.40 & 2 & 0.20 \\
\hline $\begin{array}{l}\text { Good Project } \\
\text { Management } \\
\text { Methodology }\end{array}$ & 0.10 & 1 & 0.10 & 2 & 0.20 & 3 & 0.30 & 4 & 0.40 \\
\hline $\begin{array}{l}\text { Sufficient/ } \\
\text { Acceptable } \\
\text { Operational } \\
\text { Performance }\end{array}$ & 0.10 & 1 & 0.10 & 3 & 0.30 & 2 & 0.20 & 4 & 0.40 \\
\hline $\begin{array}{l}\text { Appropriate } \\
\text { Organizational } \\
\text { Culture }\end{array}$ & 0.10 & 4 & 0.40 & 3 & 0.30 & 2 & 0.20 & 1 & 0.10 \\
\hline Totals & 1.00 & - & 2.00 & - & 2.50 & - & 2.60 & - & 2.90 \\
\hline
\end{tabular}

\section{SUMMARY AND CONCLUSION}

Applying vertical analysis to Fred David's matrix approach to strategic management decision-making, the authors created six new useful CPM matrices, the POM-CPM, M-CPM, F-CPM, HRM-CPM, R\&D-CPM, and IS-CPM. These matrices are a natural extension to the traditional CPM, whether one agrees or 
disagrees with the top ten major functional area concepts. The authors are comfortable with them, but they may be changed or adjusted. The major advantage of expanding the CPM is straightforward because the new matrices allow for greater depth of analysis in strategic management decision-making. Because of the greater contrast among companies, organizations are encouraged to apply forced rankings in these matrices, which were used by the authors when extending the original CPM concept horizontally. So what does this extra data for analysis do for analysts? Our simple conclusion is that it provides the top critical success factors for each functional business area for a more focused depth of comparison and insight to help improve the analytical effectiveness of decision-making in strategic management. Moreover, the new functional strategic matrix tools allow for more thoroughness and in-depth CPM analysis so that important data are not overlooked.

\section{REFERENCES}

Allen, M. 1979. Diagramming GE's planning for what's WATT. In Pennington, M. W., \& Allio, R. J. (Eds.) Corporate planning: Techniques and applications. New York: AMACON.

Alreemy, A., Chang, V., Walters, R., and Wills, G. 2016. Critical success factors (CSFs) for information technology governance. International Journal of Information Management, 36: 907-16.

Bygrave, W. D., \& Zacharakis, A. 2010. Entrepreneurship. New Jersey: John Wiley and Sons.

Capps, C., \& Glissmeyer, M. 2012. Extending the competitive profile matrix using internal factor evaluation and external factor evaluation matrix concepts. Journal of Applied Business Research, 28 (5): 1059-62.

Cassidy, C., Glissmeyer, M., \& Capps, C. 2013. Mapping an internal-external (IE) matrix using traditional and extended matrix concepts. Journal of Applied Business Research, 29 (5): 1-5.

Capps, C., \& Cassidy, C. 2016. Expanding the competitive profile matrix (CPM): Introducing the financial competitive profile matrix (FCPM). Academy of Strategic Management Journal, 15 (2): 9-15.

Chang, H., \& Huang, W. 2006. Application of a quantification SWOT analytical method. Mathematical and Computer Modeling, 43: 158-69.

David, F. R. 2016. Strategic management concepts and cases. (16th ed.). New Jersey: Pearson Prentice Hall. 
Denolf, J., Trienekens, J., Wognum, P., van der Vorst, J., \& Omta, S. 2015. Towards a framework of critical success factors for implementing supply chain information systems. Computers in Industry, 68: 16-26.

Fleisher, C., \& Bensoussan, B. 2003. Strategic and competitive analysis: Methods and techniques for analyzing business competition. New Jersey: Prentice Hall.

Fleisher, C., \& Bensoussan, B. 2007. Business and competitive analysis: Effective application of new and classic methods. New Jersey: FT Press.

Garrison, G., Kim, S., \& Wakefield, R. 2012. Success factors for deploying cloud computing. Communications of the ACM, 55 (9).

Huang, M-J., Chen, M-Y., \& Yieh, K. 2007. Comparing with your main competitor: The single most important task of knowledge management performance measurement. Journal of Information Sciences, 33 (4): 416-34.

Huang, S-J., Wu, M-S., \& Chen, L-W. 2013. Critical success factors in aligning IT and business objectives: A Delphi study. Total quality management, 24 (10): 1219-40. Krathu, W., Pichler, C., Xiao, G., Werthner, H., Neidhardt, J., Zapeletal, M., \& Huemer, C. 2015. Inter-organizational success factors: A cause and effect model. Information Systems e-Business Management, 13: 553-93.

Poon, P., \& Wagner, C. 2001. Critical success factors revisited: Success and failure cases of information systems for senior executives. Decision Support Systems, 30: 393-41

\section{BRIEF BIOGRAPHICAL SKETCH OF AUTHORS}

Charles J. Capps III, SPHR, is a professor of management at Sam Houston State University. He earned his D.B.A. at Louisiana Tech University, and his research interests include strategic management and human resource management.

Christopher M. Cassidy is an associate professor of management at Sam Houston State University. He earned his Ph.D. at Texas A\&M University, and his research interests include strategy implementation, organizational control systems, ethics, and pedagogical research.

Renee Gravois is associate professor of marketing at Sam Houston State University. She earned her Ph.D. at Virginia Tech, and her research interest areas include: social marketing and public policy, marketing education, and student writing.

Janis A. Warner, PMP, is associate professor of management information systems at Sam Houston State University. She earned her Ph.D. at Florida Atlantic University, and her research areas include human-computer interaction technology use, IT security from the socio-organizational perspective, project management, and pedagogical research on experiential/service learning. 